Article Type: Meeting Report

URL checked and working

DRO $\quad 9.2 .17$

Meeting report 16/1316R1

Short title: 2016 IPCC Symposium report

\title{
Report of the 13th Annual International Pachyonychia Congenita Consortium
}

\section{Symposium}

L. Rittié, ${ }^{1}$ R.L. Kaspar, ${ }^{2}$ E. Sprecher ${ }^{3}$ and F.J.D. Smith ${ }^{4}$

${ }^{1}$ Department of Dermatology, University of Michigan Medical School, Ann Arbor, MI, U.S.A.

${ }^{2}$ TransDerm Inc., 2161 Delaware Ave, Santa Cruz, CA 95060, U.S.A.

${ }^{3}$ Department of Dermatology, Tel Aviv Sourasky Medical Center, Tel Aviv, Israel

${ }^{4}$ Pachyonychia Congenita Project, Salt Lake City, UT, U.S.A.

\section{Correspondence}

Frances J.D. Smith.

E-mail: frances.smith@pachyonychia.org

Accepted for publication

23 September 2016

Funding sources

The meeting was funded by the Pachyonychia Congenita Project.

Conflicts of interest

None declared.

This is the author manuscript accepted for publication and has undergone full peer review but has not been through the copyediting, typesetting, pagination and proofreading process, which may lead to differences between this version and the Version of Record. Please cite this article as doi: $10.1111 /$ bjd.15417

This article is protected by copyright. All rights reserved 


\section{Summary}

The International Pachyonychia Congenita Consortium (IPCC) is a group of physicians and scientists from around the world dedicated to developing therapies for pachyonychia congenita, a rare autosomal dominant skin disorder. The research presented at the 13th Annual Research Symposium of the IPCC, held on 10-11 May 2016, in Scottsdale, AZ, U.S.A., is reported here.

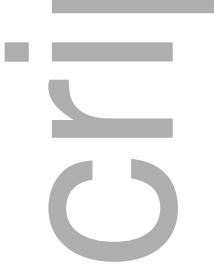

The 13th Annual Research Symposium of the International Pachyonychia Congenita Consortium (IPCC) was held in Scottsdale, AZ, U.S.A. on 10-11 May 2016. The meeting was attended by about 40 physicians and scientists from around the world devoted to discussing progress and future studies towards developing therapies for pachyonychia congenita (PC), a rare autosomal dominant keratinizing skin disorder. Severe plantar pain, palmoplantar keratoderma including calluses with underlying blisters, and variable hypertrophic nail dystrophy are the main characteristics, often accompanied by oral leucokeratosis, cysts of various types, follicular hyperkeratosis, palmoplantar hyperhidrosis and sometimes natal teeth. ${ }^{1,2} \mathrm{PC}$ is eaused by a mutation in one of five keratin genes: KRT6A, KRT6B, KRT6C, KRT16 or KRT17. ${ }^{3}$

\section{Day 1 morning session}

The first session, chaired by Laure Rittié (University of Michigan, Ann Arbor, MI, U.S.A.), opened with a presentation on 'PC genetics' (Frances Smith, PC Project) (all presentations are listed in Table S1; see Supporting Information). An update was given on patients enrolled in The International Pachyonychia Congenita Research Registry (IPCRR), established in 2004 to collect clinical and molecular data from patients with PC worldwide. It is readily accessible as patients can self-refer through the website or via a clinician referral. Within the IPCRR there are over 700 cases with genetically confirmed PC, and more than 100 different dominant mutations have been identified (no cases confirmed of recessive PC). To date, 51 cases within the IPCRR have been identified that do not have mutations in KRT6A, KRT6B, KRT6C, KRT16 or KRT17, but rather have mutations in other genes including GJB6, TRPV3, DSG1, DSP, 
KRT9, FZD6 or $A A G A B$. While these cases are not PC, the findings provide a diagnosis to these individuals who have various known disorders distinct from PC, but with some similar features. Eli Sprecher (Tel Aviv Sourasky Medical Center, Tel Aviv, Israel) discussed 'Genodermatoses and unusual modes of inheritance'. Using a number of different skin disorders as examples, including epidermolysis bullosa simplex, epidermolytic ichthyosis, familial tumoral calcinosis, autosomal recessive congenital ichthyosis and X-linked ichthyosis, he explained different modes of inheritance from monogenic and semidominant to polygenic, and the role of modifier genes.

Pierre Coulombe (Johns Hopkins University, Baltimore, MD, U.S.A.) presented 'Keratin mutation vs. pathogenesis in PC: challenges and opportunities', giving an overview of keratins and the challenges in studying associated disorders. He discussed that although very similar in sequence homology, keratins 14, 16 and 17 have very different functions, as shown by the distinct null phenotypes in mouse models. The discussion on the complex keratin ecosystem of plantar epidermis was a great introduction for a later presentation by Michelle Kerns (Johns Hopkins University). She spoke about her very recently published work, 'The role of oxidative stress and dysfunctional Nrf2 in PC-associated palmoplantar keratoderma' ${ }^{4}$ She showed that oxidative stress and dysfunctional Nrf2 act as contributors to the pathogenesis of palmoplantar keratoderma. Keratin 16 is a regulator of Nrf2 activation, and pharmacological activation of Nrf2 or modulation of oxidative stress should be further explored as possible treatments for PC. Catherine Lu (Rockefeller University, NY, U.S.A.) reported on her recent studies, 'Stem cells in the sweat glands: wound repair and regeneration'. 5 She gave an introduction to murine sweat gland biology, demonstrating the use of mouse models to answer questions that cannot easily be done using human samples. Using the mouse model as a surrogate for human disease, she investigated keratin expression in mouse sweat glands and foot epidermis to address the question: 'why mutations in PC keratins would cause hyperhidrosis?'.

Several talks throughout the meeting focused on plantar pain, the most debilitating symptom of PC (shared by $95 \%$ of patients; www.pachyonychia.org). The significant plantar pain endured by patients with PC and its effects on patients' quality of life has been a major focus of the IPCC for several years. Great strides were reported at the PC meeting. To gain a better understanding of the type of pain that patients experience, the first pain research study for PC was started in 2011, at a patient support meeting on 35 U.S. patients with mutations in KRT6A, KRT6B, KRT16 or KRT17. The study concluded that significant neuropathic pain was observed in patients with PC. ${ }^{6}$ 
Silviu Brill (Pain Medicine Institute, Tel Aviv Medical Center, Israel) presented results from a second larger study conducted on 62 patients with PC with mutations in KRT6A or KRT16 (and 45 matched controls). This study was carried out at three PC patient support meetings in 2014-2015, held in Paris, France; Edinburgh, Scotland; and Newark, U.S.A. by the same group of pain specialists, led by Ruth Defrin (Tel Aviv University, Israel). The research questions were (i) are patients with PC experiencing neuropathic pain? and (ii) is there a difference in prevalence between the genotypes? A systematic quantitative controlled study was performed using conduction and pain modulation tests, as well as pain and quality-of-life questionnaires. Overall the study showed that there were sufficient signs, such as the association of PC with moderate-to-strong chronic pain and local mechanical hyperalgesia, to indicate the presence of peripheral neuropathic pain in these patients (manuscript in preparation).

Andreas Berroth (TransDerm Inc., CA, U.S.A.) gave an update on his work studying 'The role of microRNAs in PC'. He summarized his in vitro work in which he tested the effects of the microRNAs that were found to be differentially expressed in PC-involved vs. healthy skin on keratinocyte proliferation and differentiation. His early preliminary data were discussed in the context of the known pathophysiology of PC.

The morning session closed with an enlightening talk from Dennis Roop (University of Colorado, CO, U.S.A.) on the exciting progress in his lab to develop 'Stem cell therapies for EB' (epidermolysis bullosa). His lab has developed a successful protocol for growing induced pluripotent stem (iPS) cells for therapeutic use, and is moving towards the clinical application of iPS cell-based therapy for patients with recessive dystrophic epidermolysis bullosa harbouring a particular COL7A1 mutation. Dr Roop concluded by discussing the new facility for performing the work, the Charles C. Gates Biomanufacturing Facility, at the University of Colorado. The audience wondered whether iPS cell therapy would be feasible in PC given that it is inherited in a dominant fashion. Although there were foreseeable obstacles that would need to be overcome, it was discussed that it was recently shown that mutation site-specific genome editing in iPS cells from dominant negative disorders (i.e. dominant dystrophic epidermolysis bullosa) was technically feasible. ${ }^{7}$ The group thought the approach was thus worth taking into consideration

\section{Day 1 afternoon session}

The afternoon session, chaired by Roger Kaspar (TransDerm Inc., Santa Cruz, CA, U.S.A.), began with a presentation by Jiang Chen (Stony Brook University, Stony Brook, NY, U.S.A.) 
on 'Cell to cell adhesion relative to blistering'. Dr Chen described the newly discovered role of RPGRIP1L in organizing desmosome assembly in keratinocytes. Disruption of the RPGRIPIL gene resulted in intraepidermal blistering in mice. Blocking of protein kinase $\mathrm{C}$ beta II, which is consistently upregulated in these animals, partially rescued the blistering phenotype thereby revealing a novel potential therapeutic approach for blistering diseases.

Karen Wagner (University of California Davis, CA, U.S.A.) spoke about soluble epoxide hydrolase inhibitors in her presentation 'Analgesia mediated by soluble epoxide hydrolase inhibition and epoxy fatty acid metabolites'. The current approaches to target this enzyme as a potential therapy to combat neuropathic pain were outlined. She showed how inhibition of soluble epoxide hydrolase eliminates inflammatory pain and can block neuropathic pain. Examples were shown to demonstrate the effectiveness of the enzyme in clinical conditions in animals, for example in laminitis, a severe inflammation of the hoof in horses that triggers neuropathic pain. ${ }^{8}$ She discussed how this therapy may be relevant for PC.

Birgit Lane (Institute of Medical Biology, A*STAR, Singapore) gave an overview of the growth of skin research in Singapore with the development of the Skin Research Institute of Singapore, a collaboration between the Agency for Science, Technology and Research (A*STAR), the National Skin Center and Nanyang Technological University. She discussed ongoing studies in her own lab using cell models to study epidermolysis bullosa simplex and potential treatments.

Maria Morasso (National Institutes of Health, Bethesda, MD, U.S.A.) reported on her studies identifying single-nucleotide polymorphisms present in different hair keratins expressed in tooth enamel. ${ }^{9}$ She presented data from an ongoing study in her laboratory looking at single-nucleotide polymorphisms in keratins in dental enamel from teeth collected from patients with PC to investigate whether there is any relationship between PC and dental caries. Tycho Speaker (TransDerm Inc., Santa Cruz, CA, U.S.A.) spoke about a Flex-PAD soluble microneedle array system as an alternative to hypodermic injection to administer botulinum toxin. Botulinum toxin is being considered by the IPCC for a future clinical trial to reduce the painful keratoderma (see below). The strategy takes advantage of the ability of botulinum toxin type A to suppress eccrine sweat glands, inhibiting sweat production, potentially reducing plantar blistering and pain. Several small studies have shown that injection of botulinum toxin type A into plantar calluses of patients with PC results in improvement in the painful plantar keratoderma. ${ }^{10-13}$ The speaker went on to describe and demonstrate a polyvinyl alcohol-starch film (Perspirograph) that was developed at TransDerm Inc., as an alternative to the traditional 
starch-iodine test to detect and quantify small quantities of sweat, including from individual pores.

Christopher Bunick (Yale University, New Haven, CT, U.S.A.) gave an update on his X-ray crystallography studies entitled 'Defining the chemical and structural properties of human keratins using X-ray crystallography'. His work shows the importance of structural biology and how it can explain molecular mechanisms to help link genotype with the clinical phenotype of various keratin disorders, including PC. ${ }^{14}$ Analyses performed to determine the most common chemical changes occurring in human skin disease mutations in keratins $\mathrm{K} 1 / \mathrm{K} 10, \mathrm{~K} 5 / \mathrm{K} 14$, $\mathrm{K} 6 \mathrm{a}, \mathrm{K} 6 \mathrm{~b}, \mathrm{~K} 6 \mathrm{c}, \mathrm{K} 16$ and $\mathrm{K} 17$ identified substitution of one hydrophobic residue with another hydrophobic residue as the most common clinical change type, accounting for 20-28\% of the mutations

\section{Day 2}

The second day, chaired by Eli Sprecher, focused on treatment and proposed clinical trials. Discussions centred on clinical trial plans and on potential clinical end point measurements that would best evaluate these studies. Peter Hein (Grunenthal GmbH, Aachen, Germany) gave an insight into pain, and explained how the brain (as opposed to the body or organ) produces the pain. Pain is complex and multidimensional as it is a sensory experience/event, has a conscious/cognitive component, an emotional/affective component and a motivating component/dimension. He went on to discuss setting clinical end points for pain and different ways to measure pain objectively - 'classical' pain scales (typically unidimensional), pain measurement by tools and questionnaires, and multidimensional scales. He concluded that pain is what the patient feels and there are many factors that drive variability, which may obscure true treatment effects including effectiveness of intervention, site performance, treatment history and random variability.

Michael Polydefkis (Johns Hopkins University, Baltimore, MD, U.S.A.) presented his recent findings 'Quantitative analysis of cutaneous neuroanatomy in PC patients' - a study comparing patterns of cutaneous innervation from affected and unaffected plantar skin biopsies from patients with PC and controls. The histological findings suggest that alterations in PC extend beyond keratinocytes, and may provide insights into the study of neuropathic pain in PC and potential therapeutic strategies. ${ }^{15}$

Alain Hovnanian (Inserm UMR1163 and Imagine Institute, Paris, France) showed that the mammalian target of rapamycin pathway was activated in lesional skin from four patients with 
Olmsted syndrome with mutations in TRPV3. He finished by reporting the results of using topical sirolimus for the treatment of one patient with a TRPV3 mutation.

Edel O'Toole (Barts and The London School of Medicine and Dentistry, Queen Mary

University of London, London) talked about the mechanism of action of oral retinoids and the advantages and disadvantages of different retinoids, including the newer retinoid, alitretinoin. She presented results from a study using Mattek epidermal three-dimensional cultures treated with acitretin or alitretinoin for $48 \mathrm{~h}$. mRNA levels of the keratin genes involved in PC were measured to identify which were suppressed the most. She also discussed the revised plan for a clinical trial using oral retinoids.

Details of another proposed clinical trial, botulinum toxin injections into plantar calluses, were presented by Ofir Arzi (Tel Aviv Sourasky Medical Center, Tel Aviv, Israel). As mentioned above, while not a cure, botulinum toxin $\mathrm{A}$ is being considered as a treatment to reduce the painful plantar keratoderma and improve the quality of life of patients with PC. Botulinum toxin A treatment is well tolerated and can last for several months. There followed a discussion on the trial plan itself and the end points to be used to evaluate the trial, which include a pain scale, a 6-min walk test, Patient-Reported Outcomes Measurement Information System, PC Quality of Life questionnaire, activity monitor, patient-reported weekly diaries and clinical photographs.

The final two talks focused on nails. One of the distinguishing features of PC is variable hypertrophic nail dystrophy, ranging from just a few nails affected to involvement of all 20 nails. ${ }^{1}$ This can be in the form of severely thickened nails or nails that terminate prematurely. Patients regularly file, grind or clip thickened nails. Campbell Stewart (Lake Washington Dermatology, Kirkland, WA, U.S.A.) shared the results of his study on 'The histopathological features of the nail plate in PC'. Eighteen patients with genetically confirmed PC provided 56 nail plate specimens that were processed and stained with haematoxylin and eosin and periodic acid-Schiff stain (manuscript in preparation).

Frances Smith presented findings of a small retrospective study, 'Nail removal as a PC treatment', using data collected by the IPCRR (DeKlotz et al., manuscript accepted for publication). The study looked at how many patients had chosen to have nails removed, at what age, their PC mutation and the procedure used. Patients completed an addendum questionnaire to answer questions including whether there was an overall positive outcome, any improvement in quality-of-life measures (pain, care, infections or appearance) and would they recommend nail removal to others. 
This was followed by a discussion led by Albert Bravo (Pittsfield, MA, U.S.A.) and David Hansen (University of Utah, Salt Lake City, UT, U.S.A.) to determine the benefit of permanent destruction of selected nails and whether nail removal is an underutilized treatment option for PC. Overall, it was concluded that for some individuals permanent destruction of selected nails was beneficial, but that additional studies are needed to identify the procedure to achieve the best outcomes.

\section{Conclusions}

This year's Annual Research Symposium of the IPCC revealed important progress in understanding PC pathophysiology and translation of research findings for the treatment of patients. Firstly, identifying that patients with PC experience neuropathic pain is a critical step for designing therapeutic approaches aimed at mitigating the debilitating pain that they experience. Secondly, extending the IPCRR and generating patient-based clinical data are invaluable tools to direct and prioritize future trials. Thirdly, developing tools that allow pain assessment, ambulation quantification and measurement of sweat production in patients with PC are not only necessary but will also be key to objective assessment of the clinical efficacy of potential therapeutics. All of these steps bring us a little closer to identifying efficient therapies for PC. The symposium ended with a reminder that the IPCRR data are 'A treasure trove of information on PC' and are readily available to IPCC members for studies and articles.

\section{Acknowledgments}

We thank the staff members at the PC Project for organizing the symposium.

\section{References}

1 Eliason MJ, Leachman SA, Feng BJ et al. A review of the clinical phenotype of 254 patients with genetically confirmed pachyonychia congenita. J Am Acad Dermatol 2012; 67:680-6. 2 McLean WH, Hansen CD, Eliason MJ et al. The phenotypic and molecular genetic features of pachyonychia congenita. J Invest Dermatol 2011; 131:1015-17.

3 Wilson NJ, O'Toole EA, Milstone LM et al. The molecular genetic analysis of the expanding pachyonychia congenita case collection. Br J Dermatol 2014; 171:343-55.

4 Kerns ML, Hakim JM, Lu RG et al. Oxidative stress and dysfunctional NRF2 underlie pachyonychia congenita phenotypes. J Clin Invest 2016; 126:2356-66. 
5 Lu CP, Polak L, Rocha AS et al. Identification of stem cell populations in sweat glands and ducts reveals roles in homeostasis and wound repair. Cell 2012; 150:136-50.

6 Wallis T, Poole CD, Hoggart B. Can skin disease cause neuropathic pain? A study in pachyonychia congenita. Clin Exp Dermatol 2016; 41:26-33.

7 Shinkuma S, Guo Z, Christiano AM. Site-specific genome editing for correction of induced pluripotent stem cells derived from dominant dystrophic epidermolysis bullosa. Proc Natl Acad Sci U S A 2016; 113:5676-81.

8 Guedes AG, Morisseau C, Sole A et al. Use of a soluble epoxide hydrolase inhibitor as an adjunctive analgesic in a horse with laminitis. Vet Anaesth Analg 2013; 40:440-8.

9 Duverger O, Ohara T, Shaffer JR et al. Hair keratin mutations in tooth enamel increase dental decay risk. J Clin Invest 2014; 124:5219-24.

10 Gonzalez-Ramos J, Sendagorta-Cudos E, Gonzalez-Lopez G et al. Efficacy of botulinum toxin in pachyonychia congenita type 1: report of two new cases. Dermatol Ther 2016;

29:32-6.

11 Swartling C, Vahlquist A. Treatment of pachyonychia congenita with plantar injections of botulinum toxin. Br J Dermatol 2006; 154:763-5.

12 Swartling C, Karlqvist M, Hymnelius K et al. Botulinum toxin in the treatment of sweat-worsened foot problems in patients with epidermolysis bullosa simplex and pachyonychia congenita. Br J Dermatol 2010; 163:1072-6.

13 Tariq S, Schmitz ML, Kanjia MK. Chronic foot pain due to pachyonychia congenita in a pediatric patient: a successful management strategy. A A Case Rep 2016; 6:305-7.

14 Bunick CG, Milstone LM. The x-ray crystal structure of the keratin 1-keratin 10 helix 2B heterodimer reveals molecular surface properties and biochemical insights into human skin disease. J Invest Dermatol 2017; 137:142-50.

15 Pan B, Byrnes K, Schwartz M et al. Peripheral neuropathic changes in pachyonychia congenita. Pain 2016; 157:2843-53.

\section{Supporting Information}

Additional Supporting Information may be found in the online version of this article at the publisher's website:

Table S1 Presenters and titles. 